\title{
Aplicando Design Thinking para Melhorar a Qualidade de um Aplicativo Web Móvel
}

\author{
Anna Beatriz Marques ${ }^{1}$, Emanuelle Cavalcante ${ }^{1,2}$, Luis Rivero ${ }^{1}$, Adriana Lopes ${ }^{1}$, \\ Tayana Conte ${ }^{1}$ \\ ${ }^{1}$ Grupo de Pesquisa em Usabilidade e Engenharia de Software (USES) - Instituto de \\ Computação - Universidade Federal do Amazonas (UFAM) \\ Av. Rodrigo Otávio, $n^{\circ}$ 6.200. CEP: 69077-000, Manaus - AM - Brasil \\ ${ }^{2}$ FPF Tech \\ Av. Danilo Areosa, no 1.170, Distrito Industrial, CEP 69075-351, Manaus - AM - Brasil \\ \{anna.beatriz, luisrivero, adriana, tayana\} @icomp.ufam.edu.br, \\ emanuelle.cavalcantedfpf.br
}

Resumo. Design Thinking (DT) é uma metodologia que engloba iniciativas de inovação em atividades de projeto centrado no usuário, que pode ser aplicada para proporcionar uma melhor experiência de usuário (UX). Este artigo relata uma experiência na aplicação de DT no reprojeto do aplicativo web móvel Dona Know, o que permitiu identificar oportunidades de melhoria. Foram aplicados métodos de avaliação de UX com o objetivo de comparar a nova versão do aplicativo com a versão anterior ao reprojeto. Os resultados deste trabalho demonstram que houve aumento no nivel de satisfação dos usuários ao utilizar a versão reprojetada do aplicativo.

Abstract. Design thinking (DT) is a methodology that considers innovation initiatives in user centered design activities and can be applied to improve user experience $(U X)$. This paper reports our experience when applying DT in the redesign of the Dona Know application, which allowed us to identify improvement opportunities. We applied UX evaluation methods in order to compare the new version of the application with the one before its redesign. The results from this experience show that there was as an increase in the satisfaction of the users when using the redesigned version of the application.

\section{Introdução}

A experiência do usuário (User eXperience - UX) é um conceito amplo de qualidade de uso que explora como uma pessoa se sente em relação a um produto (Tähti e Niemelä, 2006). Para que um aplicativo forneça uma boa UX, torna-se fundamental garantir a sua usabilidade e considerar as emoções dos usuários. Nesse contexto, avaliar a UX permite identificar as percepções e reações de um usuário que resultam do uso de um produto (Tähti e Niemelä, 2006). Assim, é possível identificar potenciais problemas no uso de aplicações e suas causas, bem como sugestões de melhoria para a aplicação.

Com o intuito de desenvolver aplicações com qualidade e que proporcionem uma melhor UX, o Design Thinking (DT) surge como uma metodologia orientada à resolução de problemas através de uma abordagem centrada no usuário, inovadora, 
colaborativa e experimental (Brown, 2008). O DT promove um estilo de pensamento complementar na equipe de desenvolvimento, ampliando suas habilidades de resolução de problemas com o objetivo de tornar seus resultados mais inovadores (Lindberg et al., 2011). Neste sentido, o DT proporciona a aceitação e satisfação dos usuários, sendo uma poderosa ferramenta para o projeto e reprojeto de aplicações (Fawcett et al., 2013). Porém, integrar DT com sucesso no desenvolvimento de aplicações ainda é um desafio (Lindberg et al., 2011). Assim, tornam-se necessários relatos da aplicação de DT neste contexto, bem como discutir os resultados obtidos na qualidade das aplicações, para incentivar a indústria de software a integrar DT em seu processo de desenvolvimento.

O Aplicativo Dona Know tem por objetivo listar eventos da agenda cultural da cidade de Manaus (AM). No entanto, devido aos problemas de usabilidade que este possui (Rivero et al., 2014), a metodologia de DT foi aplicada para reprojetar sua interface, com o objetivo de melhorar sua UX. Para verificar o efeito das melhorias na nova solução obtida, foi realizada uma avaliação de UX. Ao descrever a metodologia seguida, são fornecidas diretrizes e indícios para o incentivo da aplicação de DT pela indústria de software no reprojeto de aplicações. Este artigo está organizado como segue: a Seção 2 descreve a metodologia de DT. A Seção 3 apresenta o aplicativo Dona Know e a aplicação de DT em seu reprojeto. A Seção 4 mostra a avaliação de UX da nova versão do aplicativo. Por fim, a Seção 5 discute as conclusões deste trabalho.

\section{Design Thinking}

Para desenvolver produtos inovadores, técnicas criativas e de inovação como o Design Thinking (DT) vêm sendo aplicadas (Fawcett et al., 2013). Segundo Brown (2008), trata-se de uma abordagem centrada no usuário para fomentar a inovação, considerando métodos como observação, colaboração, aprendizado, prototipação de ideias e testes. Uma das vantagens da aplicação de DT é que seus conceitos podem ser aplicados em qualquer cenário e fase (Thoring e Müller, 2011), com as etapas (Vieira et al., 2014) de:

Imersão: É a primeira etapa do DT e está dividida em duas fases: (1) Empatia, que consiste em imaginar e observar cenários a partir de diversas perspectivas dos usuários com o objetivo de capturar o contexto e problemas que serão o foco do projeto (Brown, 2008), e (2) Análise e Síntese, onde são analisadas as informações do usuário e obtidos insights para propor novas soluções.

Ideação: Nesta etapa, soluções inovadoras são criadas a partir dos insights obtidos na etapa de Imersão. É quando ocorre o grande brainstorming e geralmente surgem "ideias fora da caixa" (Vieira et al., 2014). Os envolvidos são estimulados a pensar e propor soluções que estejam de acordo com o contexto observado.

Prototipagem: Esta etapa pode ocorrer em paralelo com as etapas de Imersão e Ideação, com o objetivo de validar as ideias geradas por meio de protótipos e testes. A prototipação permite a avaliação das soluções junto a uma amostra de usuários e a antecipação de problemas decorrentes do uso da solução (Brown, 2008). Técnicas de avaliação de UX podem ser aplicadas, como forma de avaliar as soluções propostas.

As etapas do DT podem ser ajustadas às necessidades do projeto e contexto do problema. Podem ainda, ser realizadas de forma incremental e em todas as etapas de DT, é possível usar abordagens para projetar, reprojetar e avaliar a UX. De Paula et al. (2014) relatam a aplicação de DT com foco na UX e usabilidade no desenvolvimento da 
aplicação Brasil +9 por alunos de graduação. A aplicação alcançou a $3^{a}$ posição na classificação dos aplicativos mais baixados no Brasil. Um relato da aplicação de DT no âmbito da Petrobras Distribuidora S.A. é descrita em Vieira et al. (2014). O DT foi aplicado no desenvolvimento de uma ferramenta de apoio à gerência do conhecimento, a Wiki BR. O resultado obtido convergiu para a elaboração de um plano de gestão de mudanças e comunicação para além de desenvolver, motivar o uso da Wiki BR. A seção seguinte apresenta a aplicação de DT no reprojeto do aplicativo Dona Know.

\section{Aplicando Design Thinking para o Reprojeto do Aplicativo Dona Know}

\subsection{O Aplicativo Web Móvel Dona Know}

Dona Know ${ }^{1}$ é um site criado em 2009 que disponibiliza uma descrição de eventos da agenda cultural da cidade de Manaus (AM). Ao observar que a maioria dos acessos ao site era realizada por dispositivos móveis, a equipe de desenvolvimento decidiu criar uma versão móvel para facilitar o acesso rápido às informações diretamente do celular, desenvolvendo assim o aplicativo móvel Dona Know.

Para identificar e corrigir problemas que afetassem a usabilidade do aplicativo Dona Know, foi realizada uma inspeção de usabilidade (Rivero et al., 2014). Três especialistas identificaram 26 problemas de usabilidade, a Figura 1 mostra um subconjunto de quatro dos problemas relatados.

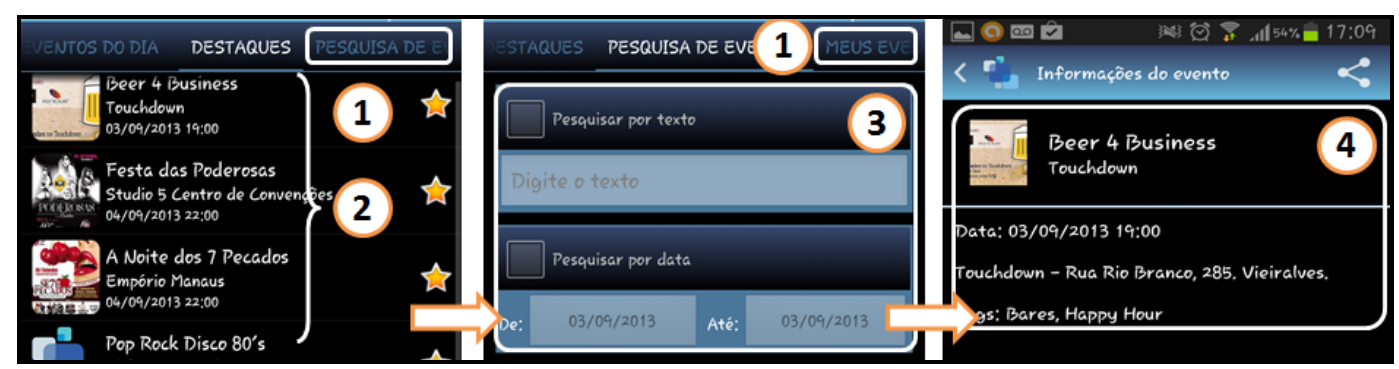

Figura 1. Principais telas da versão inicial do aplicativo Dona Know.

Na Figura 1, o problema 1 refere-se ao menu do aplicativo, que não pode ser visualizado por completo, fazendo com que um usuário inexperiente não saiba quais são as funcionalidades disponíveis, forçando-o a navegar por todas as telas para descobrilas. O problema 2 está relacionado à listagem dos itens que são muito pequenos para serem visualizados com facilidade, dificultando o uso do aplicativo. O problema 3 indica que a pesquisa de eventos não é clara devido aos termos utilizados. Finalmente, o problema 4 refere-se às informações apresentadas sobre um evento que não são suficientes, e sua organização que confunde os usuários. O DT foi aplicado para corrigir estes problemas visando obter uma solução inovadora de forma colaborativa na equipe.

\subsection{Uso do Design Thinking para o reprojeto do Aplicativo Dona Know}

O Ciclo de Design Thinking seguido no reprojeto do Dona Know foi definido a partir da seleção de técnicas aplicadas em cada etapa. Os resultados são descritos a seguir.

http://www.donaknow.com.br 
Imersão: Foram identificadas as necessidades e oportunidades de melhoria tomando como base a versão original do aplicativo Dona Know, ilustrada na Figura 1. Para isto, seis usuários potenciais do aplicativo (pessoas interessadas em obter informações sobre os eventos da cidade de Manaus) foram convidados a experimentá-lo e responder a duas perguntas em uma entrevista: (a) "O que te agradou no aplicativo?" e (b) "O que não te agradou no aplicativo?". Estas perguntas auxiliaram a identificação dos aspectos que incomodavam os usuários e dificultavam o uso do aplicativo. Para sintetizar as informações coletadas na entrevista, foi elaborada uma versão inicial de um mapa conceitual, seguindo o modelo apresentado em Bonini e Boer Endo (2011), que sugere a definição de uma frase mãe a partir da qual são criadas ramificações. Assim, foram criadas ramificações para sintetizar o que satisfaz e o que não satisfaz os usuários em relação à interface do Dona Know, apresentadas na Figura 2.

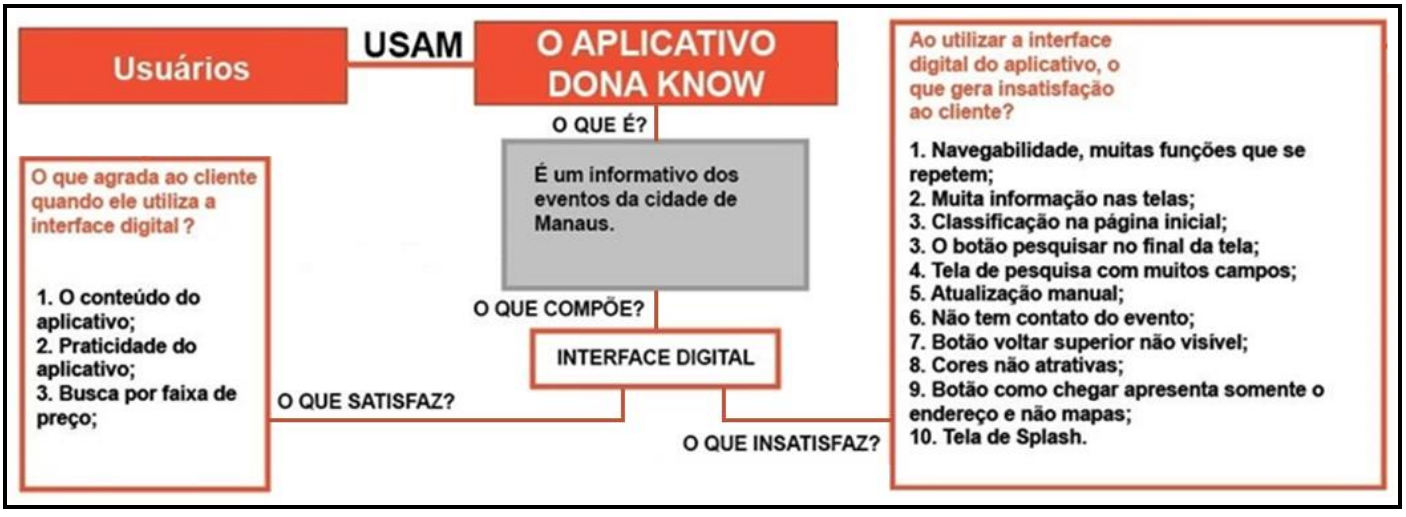

Figura 2. Parte do Mapa conceitual obtido na etapa de Imersão.

Ideação: Durante a etapa de Ideação, com base no Mapa Conceitual, foi realizado um brainstorming entre a equipe para discutir alternativas de design que poderiam minimizar o que não satisfazia os usuários até então. Nesta reunião participaram o moderador da inspeção realizada no trabalho anterior (Rivero et al., 2014) e a equipe responsável por implementar as melhorias na versão original do aplicativo (uma designer e duas analistas). Os problemas de usabilidade apontados na inspeção (Rivero et al., 2014) foram contrastados com os pontos identificados durante a imersão, para evitar que problemas de usabilidade não permanecessem no aplicativo.

Prototipação: Para avaliar e refinar as decisões de reprojeto, foram elaborados protótipos de tela implementando as decisões de design da etapa de Ideação. O moderador responsável pela inspeção anterior (Rivero et al., 2014) avaliou os novos protótipos, com o objetivo de identificar se os problemas relatados na inspeção tinham sido corrigidos ou se havia inconsistências entre as soluções de melhoria propostas e os resultados da inspeção. A Figura 3 apresenta as novas telas do aplicativo Dona Know resultantes da etapa de prototipação. Para entender melhor as modificações, estas telas serão comparadas com as telas da versão original da Figura 1.

Na Figura 3 - Tela 1, foram corrigidos os problemas de usabilidade 1 e 2 da Figura 1. $\mathrm{Na}$ entrevista, os usuários apontaram que era necessário padronizar e simplificar a interface, utilizar ícones para reduzir a quantidade de texto e aumentar a fonte do texto para maior legibilidade. Na Tela 1 foi feita uma reestruturação do aplicativo, mostrando todos os eventos na tela principal, facilitando sua filtragem por 
categorias (disponibilizadas em abas). Os eventos foram organizados segundo a data de ocorrência, criando etiquetas para indicar a data dos eventos. Para facilitar a leitura das informações, a fonte foi aumentada e apenas informações chave (nome e data) do evento foram destacadas na tela principal.
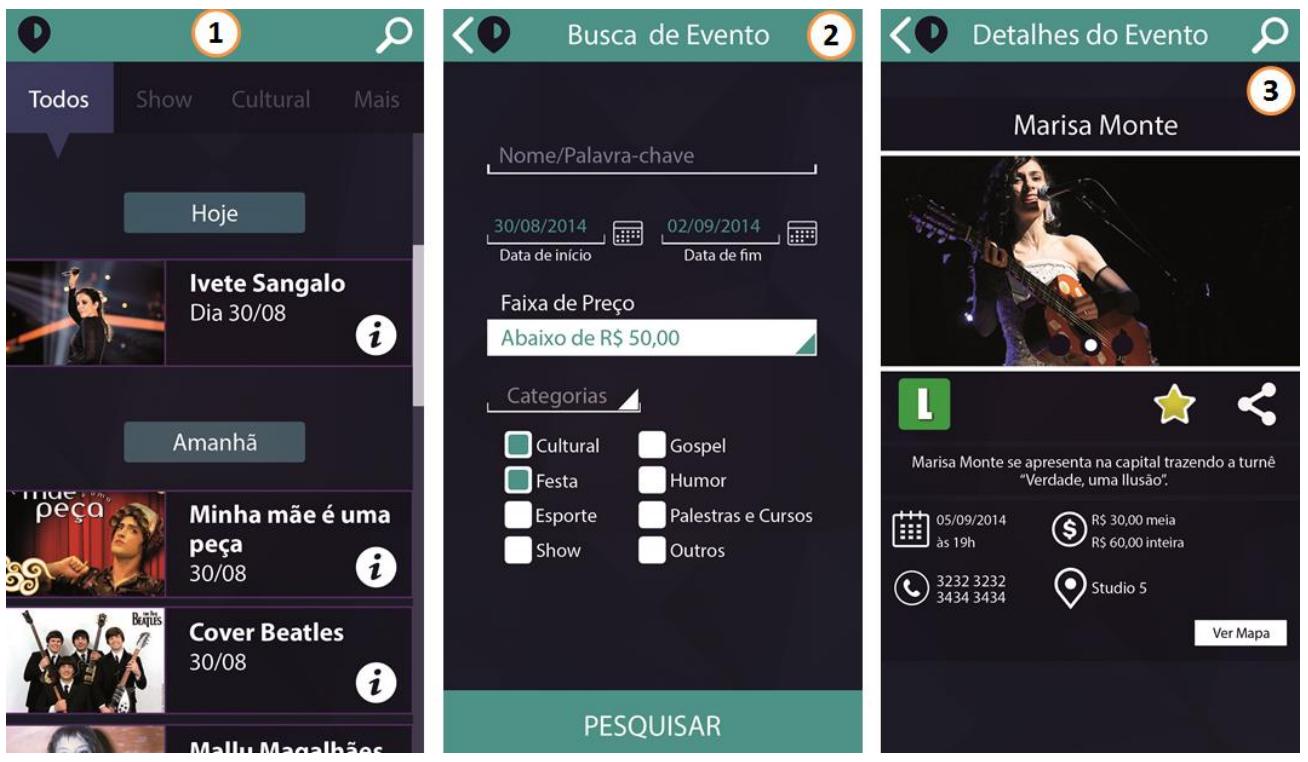

Figura 3. Principais telas da nova versão do aplicativo Dona Know.

O problema 3 na Figura 1 apontou que devido à falta de informação na tela, não era intuitivo preencher os dados da pesquisa de eventos. Para solucionar este problema e considerando que os usuários solicitaram mais opções de pesquisa (ex. busca por faixa de preço), os campos de busca foram refinados, incluindo dicas de como preenchê-los e facilitadores (ex. calendário para seleção de datas), conforme ilustra a Figura 3 - Tela 2. Em relação à Tela 3 da Figura 3, os usuários sugeriram utilizar ícones para minimizar a quantidade de texto, ampliar as figuras e texto para maior legibilidade e incluir uma funcionalidade de "como chegar?" para aumentar a utilidade do aplicativo. Ao incluir estes pontos na interface, foi corrigido o problema 4 apresentado na Figura 1 sobre as informações disponibilizadas sobre os eventos e sua legibilidade.

\section{Avaliação de UX do aplicativo Dona Know após o Reprojeto}

Com o objetivo de avaliar a UX obtida com a nova versão do Dona Know, foi realizada uma avaliação da qualidade de uso com foco na UX. Um método de baixo custo que permite avaliar e identificar fatores que afetam a UX é o Expressing Emotions and Experiences (3E). Segundo Tähti e Niemelä (2006), o método 3E disponibiliza um modelo pictórico em que o usuário pode relatar e representar: (a) o que falaria sobre o software; (b) o que está pensando sobre o software; e (c) o que está sentindo.

Para avaliar como as modificações realizadas ao aplicar DT impactaram na UX do aplicativo Dona Know, foi realizada uma avaliação de UX comparativa entre a versão inicial (Figura 1) e a versão reprojetada (Figura 3) com a aplicação do método 3E, além de perguntas sobre a utilidade, facilidade e intenção de uso do aplicativo. As etapas da avaliação foram baseadas em processos de avaliação com a participação de usuários (Tähti e Niemelä, 2006): planejamento, execução e análise de resultados. 
Durante a etapa de planejamento foram preparados os materiais para executar a avaliação, tais como o Termo de Consentimento Livre e Esclarecido (TCLE), o roteiro de avaliação e o questionário de caracterização de participantes. De forma a diminuir os custos do reprojeto, foi evitada a codificação do aplicativo antes da verificação das mudanças aplicadas. Desta forma, foram preparados protótipos de tela clicáveis de ambas as versões (original e reprojetada) do aplicativo Dona Know (criados com a ferramenta Balsamiq Mockups ${ }^{2}$ ) para serem usados durante a avaliação.

A seleção das tarefas a serem realizadas durante a avaliação (ver Figura 3) foi feita com base na importância da tarefa para atingir os objetivos de um usuário do aplicativo: (a) visualizar eventos; (b) pesquisar um evento; e (c) ver os detalhes de um evento. Para coletar dados sobre a UX e a preferência dos participantes em relação às versões do aplicativo, foi preparado um questionário de avaliação. Este questionário incluía o formulário do método $3 \mathrm{E}$ e questões sobre a utilidade, facilidade e intenção de uso (ver Figura 4). O participante também fornecia uma nota para o aplicativo (1-10) e era questionado sobre sua preferência em relação às versões do aplicativo.

A avaliação foi realizada de forma presencial, com a participação de dois moderadores, devido à possibilidade de observar os usuários durante a interação com o aplicativo. A execução da avaliação foi realizada com 15 usuários. Este número foi definido devido à disponibilidade dos usuários para participar da avaliação de forma presencial.

Ao iniciar a avaliação, o moderador entregava um celular para o participante contendo os protótipos de tela das duas versões do aplicativo. Os participantes experimentaram ambas as versões, explorando e executando as atividades definidas acima. Para evitar o viés de um usuário avaliar mais positivamente a versão reprojetada (por saber que era uma versão melhorada da versão original), ambas as versões foram apresentadas como propostas de interfaces concorrentes para o aplicativo. A ordem de apresentação foi aleatória, garantindo que metade dos usuários experimentasse primeiro a versão original e a outra metade experimentasse primeiro a versão reprojetada. Após experimentar um protótipo, o usuário preenchia o questionário avaliando sua UX.

Os resultados da análise das respostas ao questionário revelaram que a maioria $(86,67 \%)$ dos participantes preferiria utilizar a versão reprojetada do aplicativo do que a versão original $(13,33 \%)$. Estes resultados foram confirmados com relação à nota geral (de 1 a 10) fornecida pelos participantes. Em média, a versão reprojetada do aplicativo obteve uma nota de 8,7, enquanto a versão original do aplicativo obteve 7,2.

Os participantes também indicaram o seu grau de concordância com as afirmativas sobre a utilidade, facilidade e intenção de uso no questionário em uma escala de 6 itens: Discordo Totalmente, Discordo Amplamente, Discordo Parcialmente, Concordo Parcialmente, Concordo Amplamente e Concordo Totalmente. A Figura 4 apresenta o resumo das respostas dos participantes, onde mais participantes concordaram total e amplamente que a versão reprojetada era mais útil, fácil de usar e pretendiam usá-la.

\footnotetext{
http://balsamiq.com/
} 


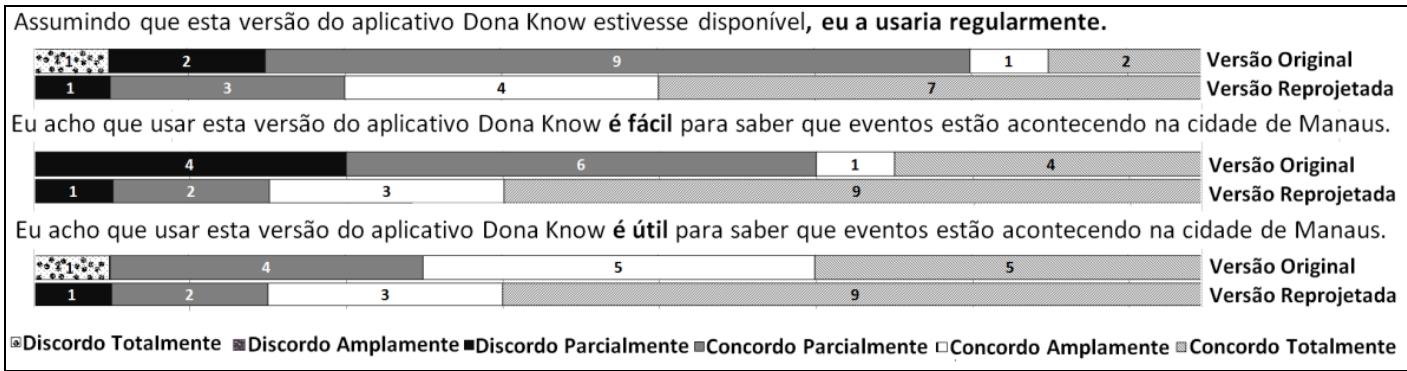

Figura 4. Resultados sobre Utilidade, Facilidade de Uso e Intenção de uso.

A Figura 5 apresenta o formulário 3E de um mesmo participante para a versão original (Parte A) e reprojetada (Parte B). Este participante teve uma experiência negativa com a versão original do aplicativo, visto que o mesmo não conseguiu realizar a pesquisa de eventos. Já na versão reprojetada, a experiência foi positiva, pois o participante considerou a pesquisa mais fácil em relação à versão original.

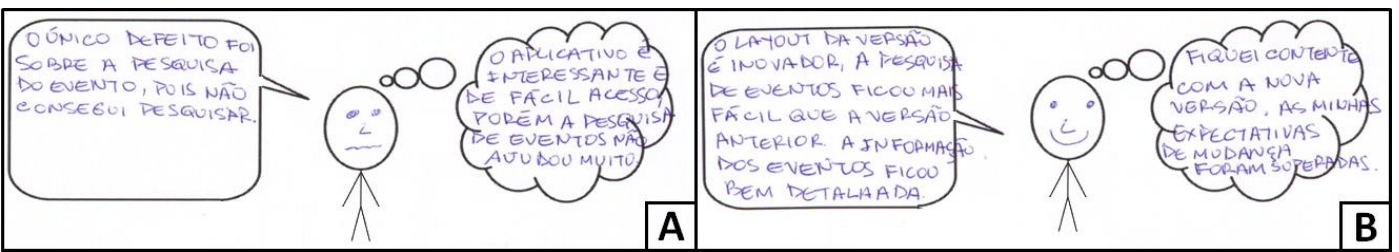

Figura 5. Exemplo de respostas do mesmo usuário para o formulário 3E sobre:

(A) versão original e (B) versão reprojetada do aplicativo Dona Know.

Para a análise dos resultados do método $3 \mathrm{E}$ foi contabilizado o número de experiências positivas e negativas e os motivos que levaram os usuários a relatá-las. Os resultados apontaram que a versão reprojetada do aplicativo despertou mais experiências positivas (11 positivas, 1 negativa e 3 neutras) do que o a versão original ( 7 positivas, 5 negativas e 3 neutras). Na versão reprojetada foram identificadas 8 pontos que impactaram na UX positivamente, tais como: "o design desta versão é melhor em relação à versão anterior" e "os detalhes são visualizados com mais clareza". Em relação à versão original, nenhum ponto positivo foi relatado pelos participantes. Estes indicaram 9 pontos que impactaram negativamente na UX, tais como: "fiquei perdido em algumas funcionalidades" e "esta versão não é explicativa e será pouco utilizada".

\section{Conclusão}

Este artigo descreveu um relato de experiência da aplicação de DT com sucesso no reprojeto do aplicativo Dona Know. A compreensão da abordagem de DT pela equipe permitiu melhorar diversos aspectos funcionais e visuais do aplicativo, visto que promoveu uma colaboração maior da equipe. As alternativas de design surgiram a partir dos pontos de melhoria identificados na etapa de imersão, e os resultados da avaliação de UX realizada apresentaram indícios da melhoria da qualidade de uso do aplicativo a partir do DT. Vale ressaltar que o reprojeto durou em torno de duas semanas, durante o qual uma nova solução foi criada, projetada e avaliada.

Neste relato de experiência, a aplicação de DT permitiu desenvolver uma nova identidade visual para aplicação. As técnicas aplicadas na etapa de imersão podem ser 
aplicadas à etapa de elicitação de requisitos de aplicações em geral. Em trabalhos futuros, seria interessante comparar diferentes formas de integração do DT no desenvolvimento de uma aplicação, no que diz respeito aos métodos e técnicas aplicadas. Além disso, o processo de avaliação de UX pode ser refinado, obtendo mais detalhes sobre a satisfação dos usuários em relação às funcionalidades do sistema individualmente. Assim, com os resultados desta e outras experiências, espera-se que a indústria de software possa integrar DT em seu processo de desenvolvimento para que seus resultados sejam mais inovadores e focados no usuário final, melhorando assim a qualidade e aceitação do software no mercado.

\section{Agradecimentos}

Os autores agradecem ao apoio financeiro fornecido a esta pesquisa pela CAPES (Coordenação de Aperfeiçoamento de Pessoal de Nível Superior) e FAPEAM (Fundação de Amparo à Pesquisa do Estado do Amazonas) através dos seguintes processos: 062.00146/2012; 062.00600/2014; e 062.00578/2014.

\section{Referências}

Brown, T. (2008) “Design thinking”, Harvard Business, Review 86, Volume 6, p. 8-92.

Bonini, L. e Boer Endo, G. (2011) "Design thinking: uma nova abordagem para inovação. Biblioteca Terra Fórum Consultores”, Biblioteca Terra Fórum Consultores.

De Paula, D., Menezes, B. e Araújo. C. (2014) "Building a Quality Mobile Application: A User-Centered Study Focusing on Design Thinking, User Experience and Usability", Design, User Experience, and Usability. User Experience Design for Diverse Interaction Platforms and Environments, A. Marcus, Switzerland, Springer, p. 313-322.

Fawcett, P., Fisher, K., Bishop, A. and Magassa, L. (2013) "Using design thinking to empower ethnic minority immigrant youth in their roles as information and technology mediaries", In Proc. of Extended Abstracts on Human Factors in Computing Systems , p. 361-366.

Lindberg, T., Meinel, C. e Wagner, R. (2011) "Design Thinking: A Fruitful Concept for IT development?", Design Thinking: Understand - Improve - Apply, Understanding Innovation. H. Plattner, C. Meinel e L. Leifer, Berlin, Springer, p. 3-18.

Rivero, L., do Valle, R. e Conte, T. (2014) "Aplicando Design e Avaliação de Usabilidade para Melhorar a Qualidade de um Aplicativo Web Móvel", Anais do Simpósio Brasileiro de Qualidade de Software (SBQS), p. 260-267.

Tähti, M. and Niemelä, M. (2006) "3e-expressing emotions and experiences" In Proc. of Workshop on Innovative Approaches for Evaluating Affective Systems. HUMAINE (Human-Machine Interaction Network on Emotion), p. 15-19.

Thoring, K. and Müller, R. (2011) "Understanding the creative mechanisms of design thinking: an evolutionary approach", In Proc. of Conf. on Creativity and Innovation in Design, p. 137-147.

Vieira, R., de Jesus Rafael, G. and da Costa, J. (2014) "Design Thinking Experience", In Anais do Simpósio Brasileiro de Fatores Humanos em Sistemas Computacionais (IHC), IHC na Prática, p. 1-4. 